\title{
Processed tomato products and risk factors for cardiovascular disease
}

\author{
Britt Burton-Freeman $^{\mathrm{a}, \mathrm{b}, *}$, Indika Edirisinghe ${ }^{\mathrm{a}}$, Jack Cappozzo $^{\mathrm{a}}$, Katarzyna Banaszewski ${ }^{\mathrm{a}}$, \\ Rose Giordano $^{\mathrm{c}}$, C. Tissa Kappagoda ${ }^{\mathrm{c}, 1}$, Yumei $\mathrm{Cao}^{\mathrm{d}}$ and Penny Kris-Etherton ${ }^{\mathrm{d}}$ \\ a Illinois Institute of Technology, Institute for Food Safety and Health, Center for Nutrition Research, \\ Bedford Park, IL, USA \\ ${ }^{\mathrm{b}}$ University of California-Davis, Department of Nutrition, Davis, CA, USA \\ ${ }^{\mathrm{c}}$ University of California-Davis, Departments of Nutrition and Internal Medicine, Davis, CA, USA \\ ${ }^{\mathrm{d}}$ Pennsylvania State University, Department of Nutritional Sciences, Chandlee Lab, University Park, \\ PA, USA
}

\begin{abstract}
.
BACKGROUND: Lipids, inflammation, oxidative stress and elevated blood pressure are targets for lowering cardiovascular disease (CVD) risk with a healthy diet.

OBJECTIVE: We tested the effect of tomato products, dietary sources of lycopene and potassium, on changes in lipids/lipoproteins, inflammation and blood pressure.

METHODS: In a two-center, randomized, parallel, controlled trial, processed tomato or non-tomato products matched in energy, sodium, and sugar content (HT = high tomato, 5 servings/day vs LT $=$ low tomato/control, $<1$ serving/day, respectively) were included in the diets of free-living overweight men and women 21 to 70 years of age $(n=53)$ for 6 weeks. All subjects had a 3-week LT run-in followed by either 6-week HT or LT/control diet. A random subset of 23 subjects had 2 postprandial (6 hour) visits at weeks 0 and 6 to assess the enduring effects of the HT vs LT diets on high fat meal-induced changes in lipids, insulin, glucose, inflammation, and blood pressure.

RESULTS: Fasting lipids/lipoproteins, insulin, glucose and C-reactive protein did not differ between the HT vs LT diets after 6 weeks intervention. Diastolic blood pressure was significantly decreased on the HT diet after 6 weeks compared to baseline, week $0(p=0.02)$ and compared to LT at week $6(p=0.03)$. Systolic blood pressure was not different between treatments at 6 weeks $(p=0.11)$. Tomato-associated effect sizes on blood pressure were greater in a sub-set of subjects with elevated blood pressure but were not statistically powered to reveal a significant effect between LT and HT treatments; however, the effect on lowering diastolic blood pressure remained, $p<0.05$ compared to baseline). Chronic intervention with processed tomatoes did not impact postprandial glucose, insulin, lipids or inflammatory marker responses to a high fat meal challenge at the end of the study $(n=23)$.

CONCLUSIONS: Processed tomatoes decreased diastolic blood pressure, but had no significant effects on other endpoints. Future research is required to confirm and potentially extend these findings in individuals with elevated blood pressure.
\end{abstract}

Keywords: Tomatoes, lycopene, cardiovascular disease, inflammation, lipids, blood pressure

\footnotetext{
${ }^{1}$ Deceased.

*Corresponding author: Britt Burton-Freeman, Illinois Institute of Technology, Institute for Food Safety and Health, Center for Nutrition Research. 6502S. Archer Rd, Bedford Park, IL 60501, USA. Tel.: +1 708341 7078; E-mail: bburton@iit.edu.
}

\section{Introduction}

The mechanisms of the cardiovascular benefits of tomatoes and lycopene are not fully understood; however, there is evidence that tomatoes and lycopene decrease risk factors for CVD [1]. Women consuming 10 or more tomato-based food products each 
week, compared with those consuming less than 1.5 servings per week had modest, but statistically significant improvements in total cholesterol, the TC:HDL cholesterol ratio, and hemoglobin A1c [2]. These epidemiological findings are supported by recent clinical trial evidence showing improvements in lipids and lipoproteins after chronic consumption of tomato products including total cholesterol [3], LDL cholesterol [3], and HDL cholesterol [4]; however, several others have shown no effect [5-7]. Similarly, effects on emerging risk factors of CVD have been documented including increased endothelial function [8], and decreased oxidized LDL [5], and inflammation [9] after chronic or acute consumption of tomato products $[10,11]$, or lycopene supplements [9]. However, the results are mixed with several studies reporting no measurable effect [12-14]. A major drawback of many of studies is lack of appropriate controls. A review examining the efficacy of lycopene supplements and tomato products on intermediate cardiovascular risk factors found variation in response profiles depending on endpoints measured, study design, comparator controls, among other factors; and highlighted the need for more research, particularly controlled trials to better understand the potential effects of tomato intake on CVD risk factors [1].

Processed tomato products contain appreciable amounts of the antioxidant lycopene, along with other nutrients associated with CVD risk reduction, such as beta-carotene, potassium and fiber [15]. The present study is a subset of a larger study exploring various outcome variables associated with CVD risk in overweight adult men and women who included tomato or non-tomato products equivalent in energy, sodium and sugar in their free-living, self-selected diets for six weeks. This report describes the effects of regular consumption of processed tomato products on fasting blood lipids/lipoproteins, inflammatory status and blood pressure. In addition, the study assessed the ability of chronic tomato consumption (background tomato diet) to minimize the metabolic, oxidative and inflammatory stresses induced by a high fat meal at the end of six weeks of high vs low tomato intake.

\section{Materials and methods}

The Human Subjects Research Committee of the University of California, Davis (UCD), California, USA and the Pennsylvania State University (PSU), University Park, Pennsylvania, USA approved the research trial. The trial was conducted at UCD and PSU in accordance with the Helsinki Declaration of 1975 as revised in 1983. All subjects signed a written informed consent form before any study-related procedures were performed. The trial is registered at ClinicalTrials.gov NCT00937742.

\subsection{Subjects}

Participants were recruited through flyers and online media outlets in Davis and Sacramento, California, and in State College/University Park, Pennsylvania areas. Each site aimed to enroll 27 subjects with the intent of having 50 subjects complete the study. Eligible subjects were adults $(21-70$ years of age) overweight or obese (BMI $25-35 \mathrm{~kg} / \mathrm{m}^{2}$ inclusive, calculated from the average of two body weight assessments in $\mathrm{kg}$ using a calibrated digital scale and the average of two height measurements in meters using a standard wall-mounted stadiometer) men and women. Exclusion criteria included: smoking (current smoker determined through survey and interview), elevated fasting glucose ( $>126 \mathrm{mg} / \mathrm{dL})$ or taking medication(s) such as lipid or blood pressure lowering, hormone replacement therapy or anti-inflammatory medications or dietary supplements that would interfere with outcomes of our study. Potential participants with known allergy or intolerance to tomatoes, or who had documented atherosclerotic disease, inflammatory disease, diabetes mellitus, or any other disease were also not eligible. Fifty-four men and women met study criteria and were enrolled in the study. Two subjects dropped out early. Twenty-six $(n=26)$ subjects participated in the acute postprandial study, of which 23 had evaluable data for the analyses.

\subsection{Study design}

This was a 2-center, 2-arm, randomized, treatmentcontrolled, parallel design human trial to evaluate the effect of chronic and acute consumption of processed tomatoes on risk factors for CVD. Participants were randomized based on a computerized randomization schedule for each site to either a high tomato intake group (HT) or a low tomato intake group (LT) for 6 weeks after a 3-week run-in period of low tomato consumption. Fasting blood samples were collected and blood pressure was measured at randomization (week 0 ) and at the end of 6 weeks intervention. Blood pressure was recorded after a 10-minute period of rest 


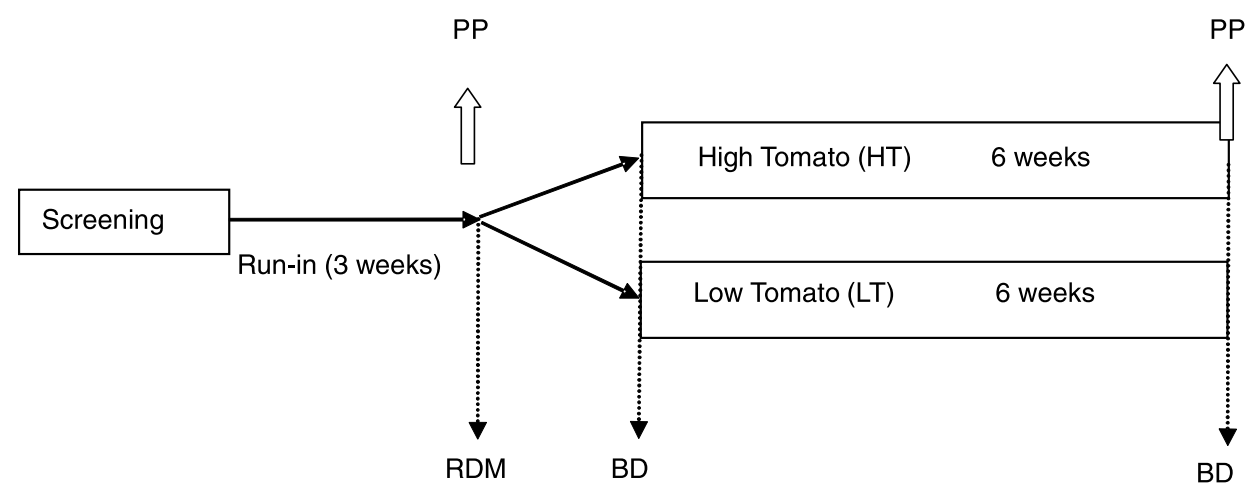

Fig. 1. Study Schema - 3 week Run-in followed by 6 weeks high tomato (HT) or low tomato (LT) diet. Fasting blood samples collected before and after chronic intervention (BD, fasting blood draw). A postprandial study (PP) of the response to a high fat meal over $6 \mathrm{~h}$ was conducted before and after respective HT or LT interventions. PP sampling times were 0 (fasting), 30, 60, 90, 120, 180, 240, 300, 360 min. RDM, Randomization.

Table 1

Foods consumed during 6-week intervention

\begin{tabular}{|c|c|c|c|c|c|c|}
\hline Food Sequences & $\begin{array}{l}\text { Serving } \\
\text { Size }\end{array}$ & $\begin{array}{l}\text { Total Dry } \\
\text { Weight }(\mathrm{g})\end{array}$ & $\begin{array}{l}\text { Lycopene } \\
\text { (mg/serv) }\end{array}$ & $\begin{array}{c}\text { B-Carotone } \\
(\mu \mathrm{g} / \text { serv })\end{array}$ & $\begin{array}{l}\text { Vitamin C } \\
(\mathrm{mg} / \mathrm{serv})\end{array}$ & $\begin{array}{c}\text { Total Phenols } \\
\text { (mg/serv) }\end{array}$ \\
\hline \multicolumn{7}{|l|}{ High Tomato $(H T)$} \\
\hline${ }^{1}$ Tomato Juice* & $1 / 2 \mathrm{c}$ & 14.8 & $33.2 \pm 1.3$ & $339 \pm 14$ & $21.5 \pm 0.2$ & $145 \pm 8$ \\
\hline${ }^{2}$ Tomato Sauce* & $1 / 2 \mathrm{c}$ & 16.8 & $37.5 \pm 0.2$ & $346 \pm 3$ & $7.5 \pm 0.1$ & $153 \pm 2$ \\
\hline${ }^{3}$ Spaghetti Sauce* & $21 / 2$ Tbsp & 17.9 & $37.0 \pm 0.0$ & $309 \pm 40$ & $8.2 \pm 0.1$ & $169 \pm 2$ \\
\hline${ }^{4}$ Diced Tomatoes* & $1 / 2 \mathrm{c}$ & 23.3 & $40.1 \pm 0.2$ & $382 \pm 8$ & $18.8 \pm 0.0$ & $207 \pm 1$ \\
\hline${ }^{5}$ Tomato Soup* & $2 / 3 c$ & 29 & $31.6 \pm 1.4$ & $339 \pm 1$ & $19.1 \pm 0.0$ & $208 \pm 8$ \\
\hline${ }^{6}$ Ketchup $+{ }^{7}$ Salsa* & 6Tbsp, 2Tbsp & 35.4 & $48.3 \pm 0.8$ & $464 \pm 3$ & $9.2 \pm 0.1$ & $215 \pm 1$ \\
\hline \multicolumn{7}{|l|}{ Low Tomato $(L T)$} \\
\hline${ }^{8}$ Yoshida's Gourmet Sauce & $21 / 2 \mathrm{Tbsp}$ & 30.7 & $0.0 \pm 0.0$ & $0.0 \pm 0.0$ & $0.0 \pm 0.0$ & $138 \pm 2$ \\
\hline${ }^{9}$ Sierra Mist $+{ }^{10}$ Table Salt & $8 \mathrm{oz}, 1 / 2 \mathrm{tsp}$ & 36.6 & $0.0 \pm 0.0$ & $0.0 \pm 0.0$ & $8.3 \pm 0.2$ & $76 \pm 2$ \\
\hline${ }^{11}$ Applesauce $+{ }^{12}$ Vegetable Broth & $1 / 2 \mathrm{c}, 11 / 2 \mathrm{c}$ & 28.5 & $0.3 \pm 0.0$ & $271 \pm 14$ & $17.9 \pm 0.2$ & $152 \pm 2$ \\
\hline
\end{tabular}

$* 5.5 \mathrm{oz}$ of tomato paste was also consumed and included in food chemistry analysis. ${ }^{1}$ Vegetable Juice, Campbells, Camden, NJ; ${ }^{2}$ Tomato Sauce, Pacific Coast Producers, Woodland, CA; ${ }^{3}$ Spaghetti Sauce, H.J. Heinz Company, Pittsburgh, PA; ${ }^{4}$ Diced Tomatoes, DelMonte Foods, San Francisco, CA; ${ }^{5}$ Tomato Soup, Campbells, Camden, NJ; ${ }^{6}$ Ketchup, H.J. Heinz Company, Pittsburgh, PA; ${ }^{7}$ Salsa, Campbells, Camden, NJ; ${ }^{8}$ Yoshida's Gourmet Sauce, H.J. Heinz Company, Pittsburgh, PA; ${ }^{9}$ Sierra Mist, Purchase, NJ; ${ }^{10}$ Table Salt, JLW Foods, Virginia Beach, VA; ${ }^{11}$ Applesauce, Mott's Inc, Rye Brook, NY; ${ }^{12}$ Vegetable Broth, Campbells, Camden, NJ.

in the sitting position with legs uncrossed and feet flat on the floor by a study designated nurse at each site who was unaware of the diet randomization. Two measurements were obtained with an interval of 5 minutes between measurements and the second measurement used for analysis. Subjects participating in the acute postprandial study consumed a non-tomato containing high fat test meal after an overnight fast at the beginning of week 1 (day 0 ) and again at week 6 ; and blood was collected at pre-designated intervals for $360 \mathrm{~min}(6 \mathrm{~h})$ at each visit. This part of the study was included to explore the potential effect of the tomato diet (background diet) to modify acute responses to a high fat-challenge meal when tomatoes are not consumed with the meal (Fig. 1).

\subsection{Experimental diets}

Participants were counseled by study dietitians throughout the trial to consume a very low/no tomato diet for 3 weeks during the run-in $(<300 \mathrm{~g} /$ week) and to incorporate study foods (Table 1) into their diets after randomization. The HT diet required participants to consume $\sim 200 \mathrm{~g} / \mathrm{d}$ or $1400 \mathrm{~g} /$ week of tomato products $(\sim 75-80 \%$ of total fruit and vegetable intake) and the LT diet required participants to consume non-tomato control foods (Table 1); matched for energy, sodium and sugar content of HT products) and limit tomato products $(<40 \mathrm{~g} / \mathrm{d}$ or $<300 \mathrm{~g} /$ week $0, \sim 15-20 \%$ of total fruit and vegetable intake). Participants also were counseled to 
Table 2

Nutrient profile of postprandial challenge meal ${ }^{1}$

\begin{tabular}{lc}
\hline & High fat challenge meal (714 kcal) \\
\hline Total Fat $(\mathrm{g})$ & 49.1 \\
SFA $(\mathrm{g})$ & 7.9 \\
MUFA $(\mathrm{g})$ & 11.7 \\
PUFA (g) & 27.4 \\
CHO $(\mathrm{g})$ & 56.3 \\
Protein $(\mathrm{g})$ & 14.3 \\
Fiber $(\mathrm{g})$ & 4.7 \\
Cholesterol (mg) & 12 \\
Sodium $(\mathrm{mg})$ & 1083 \\
\hline
\end{tabular}

${ }^{1}$ Values derived from United States Department of Agriculture Agricultural Research Service National Nutrient Database for Standard Reference Release 28, http://ndb.nal.usda.gov/ [15].

lower their total sodium intake consistent with current dietary recommendations.

In addition to the random assignment to either the HT or LT groups, participants were randomly assigned to a treatment sequence relevant to study-product distribution. Assignment to the HT intervention required all subjects to consume tomato paste $(\sim 150 \mathrm{~g} / \mathrm{d})$ plus a companion tomato product that was rotated in the diet on a weekly basis per the sequence assignment. The processed tomato companion products are shown in Table 1.

The postprandial study included the consumption of a high fat test meal comprised of a plain bagel $(35 \mathrm{~g})$, fat free cream cheese $(15 \mathrm{~g})$ and a vanilla flavored dairy cream and ice shake (Table 2). Participants in the postprandial study consumed the challenge meal for breakfast after an overnight fast and blood samples were collected at timed intervals before and after meal consumption. Blood collection occurred at 0 time (fasting) and 30 minutes and then hourly thereafter to $6 \mathrm{~h}$. All blood samples in the fasting state were collected using butterfly needles and postprandial samples were collected via indwelling catheters placed and maintained by a registered nurse. Blood samples were collected into EDTA containing tubes and immediately placed on ice and centrifuged to obtain plasma within $1 \mathrm{~h}$.

\subsection{Biochemical analysis}

Plasma from blood collections were stored at $-80^{\circ} \mathrm{C}$ until analyzed for lipids/lipoproteins, glucose, insulin, high sensitivity (hs)-C Reactive Protein (CRP), Interleukin (IL)-1 $\beta$, IL-6, tumor necrosis factor (TNF)- $\alpha$, intracellular- and vascular- adhesion molecules (ICAM and VCAM), and plasminogen activator inhibitor (PAI)-1. High sensitivity assay systems were used for the measurement of hs-CRP, IL-1 $\beta$, IL-6, TNF- $\alpha$, ICAM and VCAM (R\&D system, Minnesota, USA). PAI-1 was measured using kits purchased from American Diagnostics (Greenwich, USA). Plasma lipids/lipoproteins and glucose were analyzed using Automated Clinical analyzer (Daytona, Randox, Co. Antrim, UK) using enzymatic methods (Randox Assay kits, Antrim, UK), and insulin was measured by AlphaLisa Method (Perkin Elmer, Shelton, Connecticut, USA). Samples with greater than $10 \% \mathrm{CV}$ were repeated.

\subsection{Food chemistry}

All food product combinations were analyzed chemically for vitamin $\mathrm{C}$, lycopene, $\beta$-carotene and total phenols. Each component was analyzed using published methods [16-20]. Briefly, 0.3-0.5 g of food samples were extracted with appropriate solvents and prepared for analysis by HPLC. Ascorbic acid was detected with a UV detector at $258 \mathrm{~nm}$. Lycopene was detected at $472 \mathrm{~nm}$ and $\beta$-carotene was detected at $450 \mathrm{~nm}$. For both lycopene and $\beta$-carotene, analyses utilized YMC C30 Carotenoid columns $(4.6 \times 250 \mathrm{~mm})$. Total phenolics were measured on sample test foods according to the Folin-Ciocalteu assay as described from the method of Singleton [19]. Total phenolics were expressed as milligrams of gallic acid equivalents per gram of sample and calculated to $\mathrm{mg} /$ serving (Table 1 ).

\subsection{Statistical analyses}

Two-sample $t$-tests were used to test for demographic differences between high tomato and low tomato groups. The mixed models procedure (PROC MIXED) was used to test treatment, time, and treatment by time interactions on the concentrations of various laboratory variables and blood pressure. Repeated measurements were performed for the postprandial study. Area under the curve analysis using the trapezoidal method was used where appropriate. Gender and study center were included as fixed factors in the model. Age and BMI were included as covariates in the model. The Shapiro-Wilk (W) test for the residuals was used to test for the normality of each variable. A W statistic $>0.90$ was accepted as normally distributed. Variables not meeting these criteria were log transformed prior to analysis. TukeyKramer adjusted $\mathrm{P}$ values were used to determine 
Table 3

Screening characteristics of enrolled participants ${ }^{1}$

\begin{tabular}{|c|c|c|c|}
\hline Parameters & $\begin{array}{l}\text { All participants } \\
(n=54)\end{array}$ & $\begin{array}{l}\text { HT Group }^{2} \\
\quad(n=30)\end{array}$ & $\begin{array}{c}\text { LT Group }^{2} \\
(n=24)\end{array}$ \\
\hline Age & $44.1 \pm 13.1$ & $45.0 \pm 14.5$ & $43.0 \pm 11.3$ \\
\hline BMI $\left(\mathrm{kg} / \mathrm{m}^{2}\right)$ & $29.4 \pm 3.9$ & $29.9 \pm 4.6$ & $28.8 \pm 2.7$ \\
\hline Systolic BP (mmHg) & $118.8 \pm 14.0$ & $115.5 \pm 13.3^{*}$ & $123.0 \pm 14.0$ \\
\hline Diastolic BP (mmHg) & $78.6 \pm 8.6$ & $77.3 \pm 8.7$ & $80.2 \pm 8.3$ \\
\hline $\mathrm{TC}(\mathrm{mg} / \mathrm{dL})$ & $194.5 \pm 39.5$ & $203.8 \pm 39.7$ & $182.8 \pm 36.8$ \\
\hline LDL-C (mg/dL) & $121.2 \pm 35.3$ & $129.8 \pm 35.7^{*}$ & $110.5 \pm 32.4$ \\
\hline HDL-C (mg/dL) & $49.4 \pm 15.5$ & $50.1 \pm 15.9$ & $48.5 \pm 15.2$ \\
\hline $\mathrm{TG}(\mathrm{mg} / \mathrm{dL})$ & $119.3 \pm 63.4$ & $119.2 \pm 64.5$ & $119.4 \pm 63.3$ \\
\hline TC:HDL-C & $4.3 \pm 1.6$ & $4.5 \pm 1.6$ & $4.2 \pm 1.7$ \\
\hline Glucose (mg/dL) & $90.9 \pm 8.8$ & $92.4 \pm 9.3$ & $89.0 \pm 7.8$ \\
\hline hs-CRP (mg/L) & $2.3 \pm 2.6$ & $1.9 \pm 2.2$ & $2.7 \pm 3.0$ \\
\hline
\end{tabular}

${ }^{1}$ Data are given as means $\pm \mathrm{SD} ;{ }^{2} \mathrm{HT}$ : High Tomato diet Group; LT: Low Tomato diet Group; ${ }^{*} p<0.05$ compared to LT group.

whether the differences in the outcome variables were significant. $P \leq 0.05$ was considered statistically different and $P \leq 0.10$ indicated a trend for significance. All statistical analyses were performed using SAS for WINDOWS, release 9.1.3 (SAS Institute, Cary, North Carolina).

A sample size of 50 subjects completing the study was based on power calculations to provide sufficient statistical power $(>80 \%)$ to test the primary hypothesis of the main study, which inferred a superiority effect of frequent high tomato consumption compared to low tomato consumption on improving endothelial relaxation and platelet aggregation endpoints. For the present report which describes the results of secondary outcomes, power ranged in sufficiency depending on variance. This sample size allows for sufficient power to detect reasonable changes in total cholesterol ( $8 \%$ assuming baseline of $200 \mathrm{mg} / \mathrm{dL}$ ) and LDL cholesterol (8\% difference based on baseline of $115 \mathrm{mg} / \mathrm{dL}$ ) and estimating an SD of $\sim 10-15 \%$. A completer set of 50 subjects would also allow detection of an $8 \%$ difference in systolic blood pressure assuming mean baseline of $120 \mathrm{mmHg}$ and standard deviation of $10 \%$.

\section{Results}

\subsection{Subject characteristics}

The mean age and BMI for the group at screening ( $n=54: n=35$ women and $n=19$ men) was $44 \pm 13 \mathrm{y}$ and $29 \pm 4 \mathrm{~kg} / \mathrm{m}^{2}$, respectively. Among the group of women, nine were over the age of $50 \mathrm{y}$.
Mean screening body weight and height and fasting lipid/lipoprotein and glucose concentrations are presented in Table 3.

\subsection{Nutrient analysis of dietary intervention}

Foods consumed in study were analyzed for vitamin $C, \beta$-carotene, lycopene by HPLC and total phenols by the Folin-Ciocalteu assay (Table 1). Low and high tomato food products all contained phenols with a range of 76-215 $\mathrm{mg}$ per serving. Vitamin C was present in all food products except for the Yoshida's Gourmet Sauce. All high tomato products contained an appreciable amount of $\beta$-carotene that ranged from $309-464 \mu \mathrm{g} / \mathrm{serving}$, while the low tomato products did not contain any $\beta$-carotene except for the Applesauce + Vegetable Broth food product with $207 \mu \mathrm{g} / \mathrm{serving}$. The LT products contained no measurable lycopene while the HT products contained lycopene in the range of $31.6-48.3 \mathrm{mg} / \mathrm{serving}$. All tomato products also contained appreciable amounts of potassium [15], adding approximately $1750-1850 \mathrm{mg} / \mathrm{study}$ serving to the diet.

\subsection{Changes in fasting laboratory variables after 6 weeks of high or low tomato intake}

Daily intake of tomato products for 6 weeks did not significantly affect fasting plasma lipid/lipoprotein, glucose, or insulin concentrations or fasting markers of inflammation (hs-CRP, IL-1 $\beta$, IL-6, TNF- $\alpha$ ), fibrinolysis (PAI-1) or endothelial function (ICAM, VCAM) (Table 4). 
Table 4

Fasting concentrations at week 0 (baseline) and after 6-wk high tomato (HT) or low tomato (LT) diet ${ }^{1,2}$

\begin{tabular}{|c|c|c|c|c|}
\hline Parameters & $\begin{array}{c}\text { Baseline }^{2} \text { HT } \\
(n=28)^{3}\end{array}$ & $\begin{array}{c}\text { Baseline }^{2} \mathrm{LT} \\
(n=24)\end{array}$ & $\begin{array}{l}\text { 6-wk HT } \\
(n=26)^{4}\end{array}$ & $\begin{array}{l}\text { 6-wk LT } \\
(n=23)^{4}\end{array}$ \\
\hline $\mathrm{TC}(\mathrm{mg} / \mathrm{dL})$ & $205.7 \pm 6.8$ & $196.0 \pm 8.6$ & $198.7 \pm 7.8$ & $202.1 \pm 9.1$ \\
\hline LDL-C $(\mathrm{mg} / \mathrm{dL})$ & $115.2 \pm 6.3$ & $105.7 \pm 7.0$ & $110.0 \pm 5.4$ & $113.1 \pm 8.0$ \\
\hline HDL-C (mg/dL) & $66.5 \pm 3.6$ & $61.7 \pm 3.3$ & $61.6 \pm 3.3$ & $62.8 \pm 4.7$ \\
\hline TG (mg/dL) & $119.6 \pm 10.3$ & $139.9 \pm 17.2$ & $135.7 \pm 13.8$ & $128.3 \pm 11.9$ \\
\hline Glucose (mg/dL) & $93.2 \pm 1.0$ & $95.9 \pm 1.5$ & $91.2 \pm 1.6$ & $89.4 \pm 1.4$ \\
\hline Insulin (UI) & $7.3 \pm 0.5$ & $8.4 \pm 1.0$ & $7.6 \pm 0.8$ & $7.9 \pm 1.0$ \\
\hline hs-CRP (mg/L) & $2.0 \pm 0.4$ & $1.8 \pm 0.4$ & $1.9 \pm 0.3$ & $2.9 \pm 0.6$ \\
\hline PAI-1 (ng/mL) & $36.1 \pm 3.2$ & $35.7 \pm 3.9$ & $39.1 \pm 3.0$ & $35.4 \pm 4.6$ \\
\hline IL-6 (ng/mL) & $1.9 \pm 0.3$ & $2.0 \pm 0.3$ & $2.5 \pm 0.5$ & $1.7 \pm 0.2$ \\
\hline TNF- $\alpha(\mathrm{ng} / \mathrm{mL})$ & $1.9 \pm 0.2$ & $2.1 \pm 0.3$ & $1.9 \pm 0.2$ & $2.2 \pm 0.3$ \\
\hline ICAM (ng/mL) & $11.2 \pm 0.3$ & $11.7 \pm 0.5$ & $11.5 \pm 0.4$ & $12.0 \pm 0.5$ \\
\hline VCAM (ng/mL) & $30.5 \pm 1.4$ & $32.5 \pm 1.4$ & $31.1 \pm 1.5$ & $34.2 \pm 1.8$ \\
\hline
\end{tabular}

${ }^{1}$ Data are given as means \pm SE. ${ }^{2}$ Baseline data are means after 3 week low tomato intake run-in. ${ }^{3}$ Sample size reflects missing sample for analysis $(n=2) .{ }^{4}$ Sample size reflects early drop out/lost to follow up ( $n=1$ per group) and three subjects in the HT group that were removed from analysis due to outlier data explained by recent injury, $n=1$ or restricted protocol-specified medication use, $n=2$ prior to blood collection.

\subsection{Changes in blood pressure after 6 weeks of high or low tomato intake}

Within group mean changes from baseline to week 6 revealed a significant reduction in diastolic blood pressure after HT intake $(75.7 \pm 1.5$ to $72.5 \pm 1.3$, $p=0.02$, Table 5), but not systolic blood pressure $(114.3 \pm 2.7$ to $112.8 \pm 2.3, p>0.05)$. No within treatment differences were evident in the LT condition for systolic or diastolic blood pressure in the total group $(p>0.05$, Table 5). However, an approximate $8 \%$ difference in diastolic pressure between treatments was evident at 6 weeks $(6$ week HT: $72.5 \pm 1.3$ vs 6 week LT: $78.8 \pm 2.4, p=0.03)$. No between treatment differences were observed for systolic blood pressure ( 6 week HT: $112.8 \pm 2.3$ vs 6 week LT: $119.8 \pm 3.6, p=0.11)$. A subset analysis of subjects with elevated blood pressure (excluding normotensive subjects) showed similar trends in the data, except the effect size of the tomato intervention was increased, ie., greater \% reduction in blood pressure; although fewer people in the sample compromised power to detect a statistically significant difference between treatments (Table 5).

\subsection{Changes in postprandial laboratory variables after 6 weeks of high or low tomato intake}

No differences in postprandial insulin or glucose were observed in response to the high fat meals after 6 weeks of HT and LT background dietary interventions (data not shown). Postprandial treatment-related effects were apparent for cholesterol and triglycerides over time $(p<0.01)$; however, area under the response curve (AUC) revealed no significant effects between treatments $(p>0.05$, data not shown). Additionally, no differences between HT and LT background diets on high fat meal responses were evident for hs-CRP, TNF- $\alpha$ or IL-6 ( $p>0.05$, data not shown).

\section{Discussion}

In the present study we tested the chronic effects of processed tomato products on established and emerging risk factors for CVD. In addition, we examined the effects of chronic tomato products consumption on cardio-metabolic risk factors in an acute challenge meal setting, which did not contain tomato products. Among the recognized risk factors for CVD, we found a modest reduction in diastolic blood pressure $(p<0.05)$ after 6 weeks of tomato product inclusion in the daily diet of overweight and obese men and women, but no corresponding statistically significant reduction in systolic blood pressure $(p=0.11)$ compared to LT diet. Evidence of a 5\% reduction in LDL-cholesterol after the HT diet was noted but did not meet statistical significance. Among the metabolic and biomarkers of inflammation, no significant differences within or between treatments were observed. 
Table 5

Blood pressure at week 0 (baseline) and after 6-wk high tomato (HT) or low tomato (LT) diet ${ }^{1}$

\begin{tabular}{|c|c|c|c|c|}
\hline \multicolumn{5}{|c|}{ All Participants $(n=53)$} \\
\hline Parameters & $\begin{array}{c}\text { Baseline }^{2} \text { HT } \\
\quad(n=30)\end{array}$ & $\begin{array}{l}\text { Baseline }^{2} \text { LT } \\
\quad(n=23)^{3}\end{array}$ & $\begin{array}{c}\text { 6-wk HT } \\
(n=29)\end{array}$ & $\begin{array}{l}\text { 6-wk LT } \\
(n=23)\end{array}$ \\
\hline Systolic Blood Pressure (mmHg) & $114.3 \pm 2.7$ & $122.9 \pm 3.2$ & $112.8 \pm 2.3$ & $119.8 \pm 3.6$ \\
\hline Diastolic Blood Pressure (mmHg) & $75.7 \pm 1.5$ & $79.6 \pm 1.5$ & $72.5 \pm 1.3 \dagger^{*}$ & $78.8 \pm 2.4$ \\
\hline \multicolumn{5}{|c|}{ Pre-Htn and Htn Sub-set $(n=28)$} \\
\hline Parameters & $\begin{array}{c}\text { Baseline }^{2} \text { HT } \\
(n=13)\end{array}$ & $\begin{array}{c}\text { Baseline }^{2} \mathrm{LT} \\
\quad(n=15)\end{array}$ & $\begin{array}{c}\text { 6-wk HT } \\
(n=12)\end{array}$ & $\begin{array}{l}\text { 6-wk LT } \\
(n=14)\end{array}$ \\
\hline Systolic Blood Pressure (mmHg) & $124.4 \pm 3.8$ & $129.7 \pm 3.9$ & $118.5 \pm 3.7$ & $126.3 \pm 4.5$ \\
\hline Diastolic Blood Pressure $(\mathrm{mmHg})$ & $83.1 \pm 0.9$ & $82.4 \pm 1.8$ & $76.9 \pm 1.4 \dagger$ & $82.4 \pm 3.3$ \\
\hline
\end{tabular}

${ }^{1}$ Data are given as means \pm SE. Htn, Hypertension. ${ }^{2}$ Baseline data are means after 3 week low tomato intake run-in. ${ }^{3}$ One subject had missing blood pressure data at baseline. ${ }^{\dagger} p<0.05$ compared to baseline. ${ }^{*} p<0.05$ compared to LT group at 6 week.

In the U.S., the Joint National Committee on Prevention, Detection, Evaluation, and Treatment of High Blood Pressure identified a systolic blood pressure $<120 \mathrm{mmHg}$ and a diastolic blood pressure $<80 \mathrm{mmHg}$ as being normal [21]. Prehypertension is defined as a systolic blood pressure between 120 and $139 \mathrm{mmHg}$ and/or diastolic blood pressure between 80-89 $\mathrm{mmHg}$; blood pressures above these ranges are considered hypertensive. The present study included 26 subjects who were normotensive, 24 who were pre-hypertensive and 4 who met the criteria for hypertension. The changes in blood pressure for the total group from baseline were significantly lower for diastolic blood pressure only. Previous work has indicated that lycopene supplementation reduces systolic and diastolic blood pressure in individuals with hypertension $[22,23]$. Few studies have examined the role of tomato intake and blood pressure changes [24-2]: two in individuals with type 2 diabetes mellitus [24, 26] and one in adults 40-65 y ranging in body mass index from healthy weight to obese [25]. Only one of the three studies reported a reduction in blood pressure [24]; however raw tomatoes were the intervention and differences were reported from baseline (no control diet). Evaluation of the effects of tomato products in a sub-set of subjects in the present study (excluding individuals with blood pressure in the normal range) who had elevated blood pressure revealed a greater effect size (5\% reduction in systolic blood pressure, Table 5), but with the reduced sample size this was not significant. Effects of tomato products on diastolic blood pressure remained statistically lower from baseline with HT intervention in the sub-set of participants with elevated blood pressure. Future studies are warranted to focus on blood pressure as a primary endpoint, in a well-powered study design to address effects of tomato products in individuals with blood pressure in the hypertensive range as well as those at risk (pre-hypertension) and within the normal range. Further to this recommendation, aging imparts a significant impact on blood pressure regulation, in part due to age associated effects on the endothelium [27]. Hence, assessment of blood pressure changes across age groups relative to tomato intake would provide insight on the health promoting value of tomato intake on blood pressure over decades of life when blood pressure tends to rise due to age.

Postprandial benefits were evaluated using a high fat meal challenge paradigm at the end of the 6 week intervention period to assess residual effects of the diet. While regular intake of processed tomato products consumption did not impact the postprandial biochemical endpoints evaluated, there could be acute benefits realized when processed tomato products are consumed with a meal. We have previously reported a blunted meal-induced postprandial oxidative and inflammatory stress response when tomatoes were consumed concurrently with a high fat meal in healthy weight adults [10]. Others have shown decreased oxidative stress after tomato intake in an acute meal setting [5]. Thus, certain benefits of tomato product intake may be best realized when tomato products and their active constituents are readily available during biological stress, such as with the dietary stress of a high fat meal. Further research is needed to evaluate postprandial effects of processed tomato products on key events related to the initiation and progression of cardiovascular disease.

Strengths of the study include a robust design to investigate the chronic effects of processed tomato consumption, including residual benefits in an acute meal challenge paradigm. The study used multiple 
sites and the population was representative of a group potentially responsive to tomato products. However, this was also a limitation because of added variance with the wide age range and starting health status studied. The data however do provide insight for follow up in targeted populations. Other limitations of the study included clinical vs $24 \mathrm{~h}$ ambulatory blood pressure monitoring: the latter more appropriate for a blood pressure study. However, blood pressure in the current study was a secondary endpoint and follow up studies may include alternative methodology for primary endpoint assessment. Finally, the study was fully randomized and did not match dietary groups or sites for baseline characteristics, which may be an approach for future studies.

In summary, our findings suggest potential benefits of processed tomato products on blood pressure management in overweight middle age adults. A meta-analysis of intervention trials examining the effects of lycopene on blood pressure suggests a role for lycopene in lowering blood pressure [28]. The blood pressure lowering benefits of sodium reduction and increasing dietary potassium intake are well known [29]. This study is among the few free-living randomized controlled clinical trials assessing the effect of chronic consumption of tomato products, a rich source of lycopene and potassium, on CVD risk factors controlling for energy, sugar and salt content of processed tomato products. The results were modest, but insightful for future research on blood pressure control. Considering that physicians and dietitians may be advising against processed tomato product intake due to their sodium content, this study suggests that in the context of advice to meet sodium recommendations, adding tomato products to boost lycopene and potassium intake may be beneficial in lowering blood pressure. Given that systolic and diastolic blood pressure show heterogeneous associations across a wide range of acute and chronic cardiovascular diseases and at different ages [30], further research is warranted to better understand the role of tomato products in individuals who are at risk (pre-hypertensive) or who already have elevated blood pressure and are in the intermediate category of risk for coronary artery disease [21] where lifestyle changes are advocated as the initial therapeutic intervention. Overall, this study adds to the limited research on tomato products and CVD risk factors and suggests that, despite concerns about sodium, processed tomato products are not a food to exclude in a heart healthy diet, rather these products may be a useful dietary strategy for increasing micro- and phyto- nutrient intake to help achieve target blood pressure ranges to reduce risk of cardiovascular disease.

\section{Acknowledgments}

We thank the Tomato Products Wellness Council for funding this study. Authors have no conflicts of interest. The Tomato Products Wellness Council was not involved in the study conduct or analysis or writing of this paper. Products of processed tomato manufacturers were requested and donated for the study. Tomato products and manufacturers are listed in Table 1 .

\section{References}

[1] Burton-Freeman B, Sesso H. Whole food versus supplement: Comparing the clinical evidence of tomato intake and lycopene supplementation on cardiovascular risk factors. Adv Nutr. 2014;5:457-85.

[2] Sesso HD, Wang L, Ridker PM, Buring JE. Tomato-based food products are related to clinically modest improvements in selected coronary biomarkers in women. J Nutr. 2012;142(2):326-33.

[3] Silaste ML, Alfthan G, Aro A, Kesaniemi YA, Horkko S. Tomato juice decreases LDL cholesterol levels and increases LDL resistance to oxidation. Br J Nutr. 2007;98(6): 1251-8.

[4] Cuevas-Ramos D, Almeda-Valdés P, Chávez-Manzanera E, Meza-Arana CE, Brito-Córdova G, Mehta R, Pérez-Méndez $\mathrm{O}$, Gómez-Pérez FJ. Effect of tomato consumption on highdensity lipoprotein cholesterol level: A randomized, singleblinded, controlled clinical trial. Diabetes Metab Syndr Obes. 2013;6:263-73.

[5] Agarwal S, Rao AV. Tomato lycopene and low density lipoprotein oxidation: A human dietary intervention study. Lipids. 1998;33(10):981-4.

[6] S, Horgan GW, Duthie G. Effect of a tomato-rich diet on markers of cardiovascular disease risk in moderately overweight, disease-free, middle-aged adults: A randomized controlled trial. Am J Clin Nutr. 2012;95(5):1013-22.

[7] Collins JK, Arjmandi BH, Claypool PL, Perkins-Veazie P, Baker RA, Clevidence BA. Lycopene from two food sources does not affect antioxidant or cholesterol status of middleaged 5 adults. Nutrition Journal. 2004;3:15.

[8] Vilahur G, Cubedo J, Padro T, Casani L, Mendieta G, Gonzalez A, Badimon L. Intake of cooked tomato sauce preserves coronary endothelial function and improves apolipoprotein A-1 and apolipoprotein $\mathrm{J}$ protein profile in high-density lipoproteins. Transl Res. 2014;S1931-5244(14):00420-4.

[9] McEneny J, Wade L, Young IS, Masson L, Duthie G, McGinty A, McMaster C, Thies F. Lycopene intervention reduces inflammation and improves HDL functionality in moderately overweight middle-aged individuals. J Nutr Biochem. 2013;24(1):163-8. 
[10] Burton-Freeman B, Talbot J, Park E, Krishnankutty S, Edirisinghe I. Protective activity of processed tomato products on postprandial oxidation and inflammation: A clinical trial in healthy weight men and women. Mol Nutr Food Res. 2012;56(4):622-31.

[11] Ghavipour M, Saedisomeolia A, Djalali M, Sotoudeh G, Eshraghyan MR, Moghadam AM, Wood LG. Tomato juice consumption reduces systemic inflammation in overweight and obese females. Br J Nutr. 2013;109(11):2031-5.

[12] Thies F, Masson LF, Rudd A, Vaughan N, Tsang C, Brittenden J, Simpson WG, Duthie S, Horgan GW, Duthie G. Effect of a tomato-rich diet on markers of cardiovascular disease risk in moderately overweight, disease-free, middle-aged adults: A randomized controlled trial. Am J Clin Nutr. 2012;95(5): 1013-22.

[13] Denniss SG, Haffner TD, Kroetsch JT, Davidson SR, Rush JW, Hughson RL. Effect of short-term lycopene supplementation and postprandial dyslipidemia on plasma antioxidants and biomarkers of endothelial health in young, healthy individuals. Vasc Health Risk Manag. 2008; 4(1):213-22.

[14] Markovits N, Ben Amotz A, Levy Y. The effect of tomatoderived lycopene on low carotenoids and enhanced systemic inflammation and oxidation in severe obesity. Isr Med Assoc J. 2009;11(10):598-601.

[15] United States Department of Agriculture Agricultural Research Service National Nutrient Database for Standard Reference Release 28, http://ndb.nal.usda.gov/.

[16] Doner LW, Hicks KB. High-performance liquid chromatographic separation of ascorbic acid, erythorbic acid, dehydroascorbic acid, dehydroerythorbic acid, diketogulonic acid, and diketogluconic acid. Analytical Biochemistry. 1981;115(1):225-30.

[17] Cappozzo JC, Tiong T, Hruskovich C. Quantitative HPLC Determination of Vitamin C in Meats, Fruits and Vegetables. 108th Annual AOAC International Meeting. Portland, OR, 1994.

[18] Khachic F, Goli MB, Beecher GR, Holden J, Lusby WR, Tenorio MD, Barrera MR. Effect of food preparation on qualitative and quantitative distribution of major carotenoid constituents of tomatoes and several green vegetables. J Ag Food Chemistry. 1992;40:390-8.

[19] Singleton VL, Orthofer R, Lamuela-Raventos RM. Analysis of total phenols and other oxidation substrates and antioxidants by means of the folin-ciocalteu reagent. Methods of Enzymol. 1999;299:152-78.

[20] Van Breenan RB. Innovations in carotenoid analysis using LC/S. Analytical Chemistry. 1996;68(9):299-304.
[21] James PA, Oparil SO, Carter BL, Cushman WC, DennisonHimmelfarb C, Handler J, Lackland DT, LeFevre ML, MacKenzie TD, Ogedegbe L, Smith SC, Svetkey LP, Taler SJ, Townsend RR, Wright Jr JT, Narva AS, Ortiz E. Evidencebased guideline for the management of high blood pressure in adults. Report from the panel members appointed to the Eighth Joint National Committee (JNC8). JAMA. 2014; 311(5):507-20.

[22] Paran E, Novack V, Engelhard YN, Hazan-Halevy I. The effects of natural antioxidants from tomato extract in treated but uncontrolled hypertensive patients. Cardiovasc Drugs Ther. 2009;23(2):145-51.

[23] Engelhard YN, Gazer B, Paran E. Natural antioxidants from tomato extract reduce blood pressure in patients with grade-1 hypertension: A double-blind, placebo-controlled pilot study. Am Heart J. 2006;151(1):100.

[24] Shidfar F, Froghifar N, Vafa M, Rajab A, Hosseini S, Shidfar S, Gohari M. The effects of tomato consumption on serum glucose, apolipoprotein B, apolipoprotein A-I, homocysteine and blood pressure in type 2 diabetic patients. Int J Food Sci Nutr. 2011;62:289-94.

[25] Thies F, Masson LF, Rudd A, Vaughan N, Tsang C, Brittenden J, Simpson WG, Duthie S, Horgan GW, Duthie G. Effect of a tomato-rich diet on markers of cardiovascular disease risk in moderately overweight, disease-free, middle-aged adults: A randomized controlled trial. Am J Clin Nutr. 2012;95(5): 1013-22.

[26] Upritchard JE, Sutherland WH, Mann JI. Effect of supplementation with tomato juice, vitamin $\mathrm{E}$, and vitamin $\mathrm{C}$ on LDL oxidation and products of inflammatory activity in type 2 diabetes. Diabetes Care. 2000;23:733-8.

[27] Edirisinghe I, Burton-Freeman B. Aging associated endothelial function: Role of Oxidative stress, Inflammation and Western Diet. Nutrition and Aging, 2014;2(4):197-212.

[28] Ried K, Fakler P. Protective effect of lycopene on serum cholesterol and blood pressure: Meta-analyses of intervention trials. Maturitas. 2011;68(4):299-310.

[29] Whelton PK, He J. Health effects of sodium and potassium in humans. Curr Opin Lipidol. 2014;25(1):75-9. doi: 10.1097

[30] Rapsomaniki E, Timmis A, George J, Pujades-Rodriguez M, Shah AD, Denaxas S, White IR, Caulfield MJ, Deanfield JE, Smeeth L, Williams B, Hingorani A, Hemingway $\mathrm{H}$. Blood pressure and incidence of twelve cardiovascular diseases: Lifetime risks, healthy life-years lost, and age-specific associations in 1.25 million people. Lancet. 2014;383(9932):1899-911. doi: 10.1016 